\title{
Interaction-sensitive oscillations of dark solitons in trapped dipolar condensates
}

\author{
T. Bland, ${ }^{1}$ K. Pawłowski, ${ }^{2}$ M. J. Edmonds, ${ }^{1}$ K. Rzążewski, ${ }^{2}$ and N. G. Parker ${ }^{1}$ \\ ${ }^{1}$ Joint Quantum Centre Durham-Newcastle, School of Mathematics and Statistics, Newcastle University, Newcastle upon Tyne, \\ NE1 7RU, United Kingdom \\ ${ }^{2}$ Center for Theoretical Physics, Polish Academy of Sciences, Al. Lotników 32/46, 02-668 Warsaw, Poland
}

(Received 7 October 2016; revised manuscript received 27 March 2017; published 26 June 2017)

\begin{abstract}
Thanks to their immense purity and controllability, dipolar Bose-Einstein condensates are an exemplar for studying fundamental nonlocal nonlinear physics. Here we show that a family of fundamental nonlinear wavesthe dark solitons-are supported in trapped quasi-one-dimensional dipolar condensates and within reach of current experiments. Remarkably, the oscillation frequency of the soliton is strongly dependent on the atomic interactions, in stark contrast to the nondipolar case. Established analytical techniques are shown to not capture the simulated dynamics. These sensitive waves may act as mesoscopic probes of the underlying quantum matter field.
\end{abstract}

DOI: 10.1103/PhysRevA.95.063622

Dark solitons are the fundamental nonlinear excitations of one-dimensional medium with defocusing nonlinearity, appearing as traveling localized reductions in the field amplitude. Since first realized in optical fibers [1-3], they have been observed across plasmas [4,5], water [6], magnetic films [7], and atomic Bose-Einstein condensates (BECs) [8-20]. The last system provides a commanding playground for exploring soliton physics in which the nonlinearity (viz. atomic interactions) can be precisely controlled in amplitude, time, and space [21], and almost arbitrary potentials can be painted [22]. Experiments have studied a host of fundamental properties, including their collisions $[15,16]$, creation $[8,9,12,13,17]$, interaction with impurities [20], and decay $[11,18]$. Moreover, these "quantum canaries" are touted as sensitive probes of the mesoscale quantum physics within the quantum degenerate gas [23].

It is remarkable that the dark soliton, a collective excitation, behaves to first order as a classical particle with negative effective mass, acting under the external potential [24,25]. For example, in harmonically trapped BECs, the soliton oscillates at a characteristic ratio $\omega / \sqrt{2}$ of the trap frequency $\omega$ [26-35], as confirmed experimentally [15]. This robust result, insensitive to the microscopic atomic interactions, is a signature of matter-wave dark solitons. Here we establish the form and dynamics of these fundamental structures in trapped BECs featuring dipole-dipole atomic interactions. Remarkably, the oscillations become strongly dependent on the strength and polarization of the dipolar interactions, and an extended, nonlocal characteristic is evident. We establish these solutions and their oscillatory behavior, based on one- (1D) and three-dimensional (3D) mean-field models, and demonstrate that they are accessible to current experiments.

The last decade has seen a surge of research on dipolar BECs, as realized through the condensation of vapors of $\mathrm{Cr}$ $[36,37]$, Dy [38,39], and Er [40,41] atoms. On top of the usual van der Waals (vdW) interatomic interactions, which

Published by the American Physical Society under the terms of the Creative Commons Attribution 4.0 International license. Further distribution of this work must maintain attribution to the author(s) and the published article's title, journal citation, and DOI. are isotropic and short range, the atoms possess significant magnetic dipole moments and experience dipole-dipole (DD) interactions, which are anisotropic and long range [42]. This has opened the door to studying the interplay of magnetism with quantum coherence, and local with nonlocal nonlinearities, at the control of atomic physics. Rich phenomena have been revealed, including recent observations of the quantum analog of the ferrofluid Rosensweig instability [43,44] and self-bound three-dimensional droplets $[41,45]$.

We consider a trapped, weakly interacting BEC of atoms with mass $m$ and permanent magnetic dipole moment $\mu$, polarized in a common direction, and in the limit of zero temperature. The atom-atom interactions can be approximated by the universal pseudopotential [42]

$$
U\left(\mathbf{r}-\mathbf{r}^{\prime}\right)=\frac{4 \pi \hbar^{2} a_{s}}{m} \delta\left(\mathbf{r}-\mathbf{r}^{\prime}\right)+\frac{\mu_{0} \mu^{2}}{4 \pi} \frac{1-3 \cos ^{2} \Theta}{\left|\mathbf{r}-\mathbf{r}^{\prime}\right|^{3}}
$$

The first term describes the vdW interactions, characterized by the $s$-wave scattering length $a_{s}$; this is experimentally tunable through Feshbach resonances under external magnetic or optical fields [46]. The second term is the DD interaction, where $\mu_{0}$ is the permeability of free space and $\Theta$ is the angle between the interatom vector and the polarization direction. It is useful to define the dipolar length scale as $a_{\mathrm{dd}}=m \mu_{0} \mu^{2} / 12 \pi \hbar^{2}$. The magic angle $\Theta_{m} \approx 54^{\circ}$, for which this term reduces to zero, is the crossover from attractive to repulsive DD interactions. For $\Theta>\Theta_{m}$ the dipoles repel while for $\Theta<\Theta_{m}$ they attract. The regime of "antidipoles", $\mu^{2}<0$, is accessible by tilting and rapidly rotating the polarization direction [47]; then this angular behavior becomes reversed. We quantify the interactions through the relative interaction parameter $\varepsilon_{\mathrm{dd}}=a_{\mathrm{dd}} / a_{s}$ [42], where the full range $-\infty<$ $\varepsilon_{\mathrm{dd}}<\infty$ is experimentally accessible.

The trapping potential is assumed to be harmonic and axisymmetric, $V=m\left[\omega_{z}^{2} z^{2}+\omega_{\perp}^{2} r^{2}\right] / 2$, where $\omega_{z}$ and $\omega_{\perp}$ are the axial and radial trap frequencies, respectively. The polarization is at an angle $\theta$ to the $z$ axis (this is different to $\Theta$ above, which is the polarization angle relative to the vector between two dipoles). The BEC is described by a (complex) mean-field wave function $\Psi(\mathbf{r}, t)$, normalized to the number of atoms, $N$, which obeys the dipolar Gross-Pitaevskii equation 
(GPE) [42],

$$
\begin{aligned}
i \hbar \frac{\partial \Psi}{\partial t}= & {\left[-\frac{\hbar^{2}}{2 m} \nabla^{2}+\frac{m}{2}\left(\omega_{z}^{2} z^{2}+\omega_{\perp}^{2} r^{2}\right)+\frac{4 \pi \hbar^{2} a_{s}}{m}|\Psi|^{2}\right.} \\
& \left.+\int U_{\mathrm{dd}}\left(\mathbf{r}-\mathbf{r}^{\prime}\right)\left|\Psi\left(\mathbf{r}^{\prime}, t\right)\right|^{2} d \mathbf{r}^{\prime}\right] \Psi,
\end{aligned}
$$

where $U_{\mathrm{dd}}$ denotes the DD term in Eq. (1). In effect, the BEC experiences an effective potential comprising of the static external potential, a local potential proportional to the atomic density arising from $\mathrm{vdW}$ interactions, and a nonlocal potential arising from the DD interactions.

Since dark solitons are dimensionally unstable in 3D (decaying into vortical structures via the snake instability), we focus on highly elongated BECs. First, for simplicity, we work in this quasi-1D limit $\left(\omega_{z} \ll \omega_{\perp}\right.$ and $\hbar \omega_{\perp} \gtrsim \mu$, where $\mu$ is the BEC chemical potential). The $3 \mathrm{D}$ wave function $\Psi$ then approximates the form $\Psi(\mathbf{r}, t)=\psi_{\perp}(x, y) \psi(z, t)$, where $\psi_{\perp}(x, y)=\left(l_{\perp} \sqrt{\pi}\right)^{-1} \exp \left\{-\left(x^{2}+y^{2}\right) / 2 l_{\perp}^{2}\right\}$ is the transverse ground harmonic oscillator state with characteristic length $l_{\perp}=\sqrt{\hbar / m \omega_{\perp}}$. Integrating out the transverse mode leads to an effective 1D dipolar GPE [48,49], equivalent to Eq. (2) under the replacements $\mathbf{r} \mapsto z, \Psi \mapsto \psi, a_{s} \mapsto a_{s} / 2 \pi l_{\perp}^{2}$ and

$$
U_{\mathrm{dd}} \mapsto U_{0}\left[2 u-\sqrt{2 \pi}\left(1+u^{2}\right) e^{u^{2} / 2} \operatorname{erfc}\left(\frac{u}{\sqrt{2}}\right)+\frac{8}{3} \delta(u)\right],
$$

where $u=\left|z-z^{\prime}\right| / l_{\perp}$ and $U_{0}=\mu_{0} \mu^{2}(1+3 \cos 2 \theta) / 32 \pi l_{\perp}^{3}$.

In the absence of dipoles and axial trapping, and for repulsive vdW interactions $\left(a_{s}>0\right)$, the 1D dipolar GPE reduces to the 1D defocusing cubic nonlinear Schrödinger equation. This is completely integrable, supporting a family of dark soliton solutions [24,50] with characteristic density depression and phase slip. Axial trapping and/or dipolar interactions break this integrability but continue to support dark solitons (defined broadly) which may be found numerically [25,26,51-53]. Bright [54-58] and bright-dark [59] solitons have been predicted in dipolar BECs, although these are quite distinct from dark solitons.

We can specify a criterion for a dark soliton to exist in the dipolar BEC. Within the local density approximation, the interaction terms in the 1D dipolar GPE reduce to $\hbar \omega_{\perp}\left[2 a_{s}-a_{\mathrm{dd}}(1+3 \cos 2 \theta) / 2\right] n(z)$, where $n(z)=|\psi|^{2}$ is the axial density profile. Enforcing these net interactions to be repulsive (positive) leads to the rudimentary criterion to support dark solitons,

$$
a_{\mathrm{eff}}=a_{s}\left[1+\frac{\varepsilon_{\mathrm{dd}}}{2}\left(1-3 \cos ^{2} \theta\right)\right]>0,
$$

where $a_{\text {eff }}$ is an effective $s$-wave scattering.

We illustrate the dark soliton solutions using the case of dipoles polarized perpendicular to the $z$ axis $(\theta=\pi / 2)$. The criterion (3) then reduces to $a_{s}\left(1+\varepsilon_{\mathrm{dd}} / 2\right)>0$, or, in terms of $\varepsilon_{\mathrm{dd}}, \varepsilon_{\mathrm{dd}}>-2$ for $a_{s}>0$ and $\varepsilon_{\mathrm{dd}}<-2$ for $a_{s}<0$. We only consider the solutions in these ranges; stable dark solitons require positive chemical potential $\mu>0$, the boundary for which remains close to the criteria specified based on the local density approximation, Eq. (3). Numerically it can be shown that larger values of the scattering length bring the boundary closer to $\varepsilon_{\mathrm{dd}}=-2$, whereas larger $\sigma$ pushes this boundary to smaller values of $\varepsilon_{\mathrm{dd}}<-2$. Figure 1(a) maps the density $n(z)$ (a)

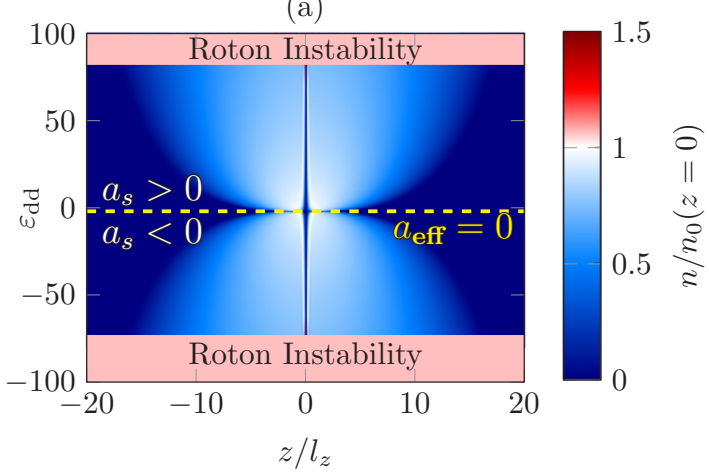

(b)

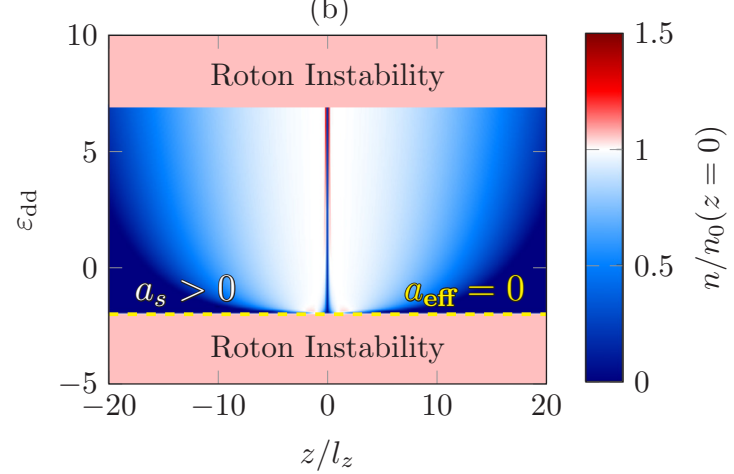

(c)

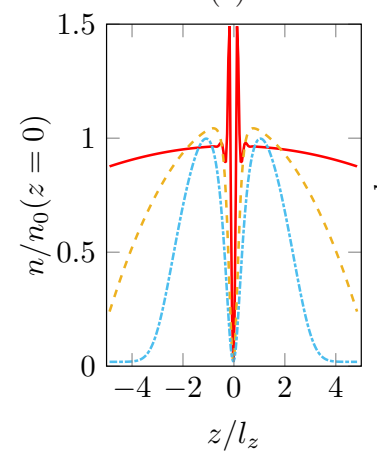

(d)

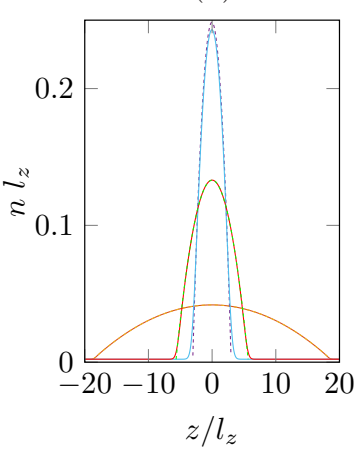

FIG. 1. Density profile $n(z)$ of the quasi-1D dipolar BEC (polarization perpendicular to the axis) featuring a central black soliton, as a function of $\varepsilon_{\mathrm{dd}}$. The vdW interactions satisfy (a) $|\beta|=61$ and (b) $\beta=1500$, and the trap frequency ratio $\omega_{z} / \omega_{\perp}=0.0025$. Only the regimes satisfying Eq. (3) are shown, with the line $a_{\text {eff }}=0$ indicated (yellow dashed line). The color scale is normalized to the peak density of the soliton-free BEC, $n_{0}(z=0)$. The roton-unstable regions extend to $\varepsilon_{\mathrm{dd}}= \pm \infty$. (c) Example density profiles, for $\varepsilon_{\mathrm{dd}}=-1.7$ (blue dot dashed line), $\varepsilon_{\mathrm{dd}}=-74$ (red solid line), and $\varepsilon_{\mathrm{dd}}=0$ (yellow dashed line). (d) Soliton-free density profile (solid lines), with the TF prediction of Eq. (4) overlaid (dotted), for the same $\varepsilon_{\mathrm{dd}}$ values as in (c).

of the quasi-1D BEC featuring a central black soliton, as a function of $\varepsilon_{\mathrm{dd}}$. The vdW interactions, characterized by the dimensionless parameter $\beta=a_{s} N l_{z} / l_{\perp}^{2}$ with $l_{z}=\sqrt{\hbar / m \omega_{z}}$ the axial harmonic oscillator length, are fixed in amplitude throughout to two characteristic values: a low value $|\beta|=61$, chosen to show stable solutions for $\varepsilon_{\mathrm{dd}}<-2$, and a larger value of $\beta=1500$ where this region is roton unstable. This black soliton state corresponds to the first excited state of the 
BEC [25], and is obtained by numerical integration of the 1D dipolar GPE in imaginary time (using a Crank-Nicolson scheme) subject to a $\pi$-phase step at the origin.

The background BEC widens as $\varepsilon_{\mathrm{dd}}$ is varied away from the line $a_{\text {eff }}=0$, caused by magnetostriction in the playoff between the vdW and DD interactions. This can be accounted for within the Thomas-Fermi (TF) approximation, valid for strong repulsive interactions and based on neglecting density gradients. Generalizing previous derivations of the ThomasFermi profile of the quasi-1D trapped BEC $[60,61]$ to include dipoles aligned at an arbitrary angle $\theta$ leads to the ThomasFermi density [62],

$$
n_{\mathrm{TF}}(z)=\frac{\left(l_{\perp} R_{z} / 2 l_{z}^{2}\right)^{2}}{a_{\mathrm{eff}}}\left[1-\frac{z^{2}}{R_{z}^{2}}\right],
$$

for $z \leqslant R_{z}$, and $n_{\mathrm{TF}}=0$ elsewhere, where $R_{z}=$ $\left[3 a_{\mathrm{eff}} N l_{z}^{4} / l_{\perp}^{2}\right]^{1 / 3}$ defines the Thomas-Fermi half-width of the BEC. The angular dependence is intuitive: for axially polarized dipoles (perpendicularly polarized), $R_{z}$ is smaller (larger) than the nondipolar case, consistent with a head-to-tail (side-by-side) alignment shrinking (enlarging) the axial extent of the cloud. The TF prediction typically agrees very well with the true profiles [see Fig. 1(d)], with significant deviations only when the net local interactions become small $\left(a_{\mathrm{eff}} \rightarrow 0\right)$.

The background BEC suffers the roton instability (RI). A trapped dipolar BEC can develop a roton (finite-momentum) minimum in its excitation spectrum which, for certain parameters, can touch zero energy, triggering an instability at finite momentum [63]. Our quasi-1D BEC has three RI regimes. The first also arises in the uniform system, as mapped out elsewhere [51,52]; e.g., in Fig. 1(a) this occurs for $\varepsilon_{\mathrm{dd}} \gtrsim-2$ with $a_{s}<0$. Two further RI regimes arise for large $\left|\varepsilon_{\mathrm{dd}}\right|$ [red bands in Figs. 1(a) and 1(b)]. For increasing magnitude of $\beta$, which can be achieved through a larger atom number, scattering length, or considering a more 3D system (increasing trap ratio $\omega_{z} / \omega_{\perp}$ ), these RI bands encroach toward $\varepsilon_{\mathrm{dd}}=0$. Hence, in Fig. 1(b) there are no stable solutions for $a_{s}<0(\beta=-1500)$.

The black soliton appears as a density notch at the origin, set upon the background BEC. For $\varepsilon_{\mathrm{dd}}=0$ and with $a_{s}>0$ the numerical solution [yellow dashed line in Fig. 1(c)] closely approximates the product of the exact black soliton solution in a uniform system [24,50] and the background density $n_{\mathrm{b}}(z)$, i.e., $n(z)=n_{\mathrm{b}}(z) \tanh ^{2}(z / \xi)$, where $\xi=1 / \sqrt{4 \pi n_{0} a_{s}}$ is the healing length at the BEC center. For $\varepsilon_{\mathrm{dd}} \neq 0$, and away from $a_{\text {eff }}=0$ and the RIs, this approximate form holds, with $a_{s}$ replaced by $a_{\text {eff }}$. However, close to a RI the dark soliton develops distinctive peripheral density ripples [e.g., red solid line in Fig. 1(c)], due to the mixing of the roton mode into this state, and as reported in the uniform system [51-53]. Meanwhile, as $a_{\mathrm{eff}}=0$ is approached, the soliton broadens while the background BEC shrinks. While we have focused on $\theta=\pi / 2$, the behavior is qualitatively similar for all $\theta>\theta_{\mathrm{m}}$ (where $\theta_{\mathrm{m}}$ is the magic angle relative to the $z$ axis), albeit with shifts in $a_{\text {eff }}$ [according to Eq. (3)] and the onsets of the RI. Meanwhile, for $\theta<\theta_{\mathrm{m}}$ the dependence on $\varepsilon_{\mathrm{dd}}$ is effectively flipped [51,52].

Next we study the oscillation dynamics of the dark soliton from the initial condition of an off-center black soliton at $z_{0}=$ $0.5 l_{z}$, although our findings are insensitive to the initial offset.

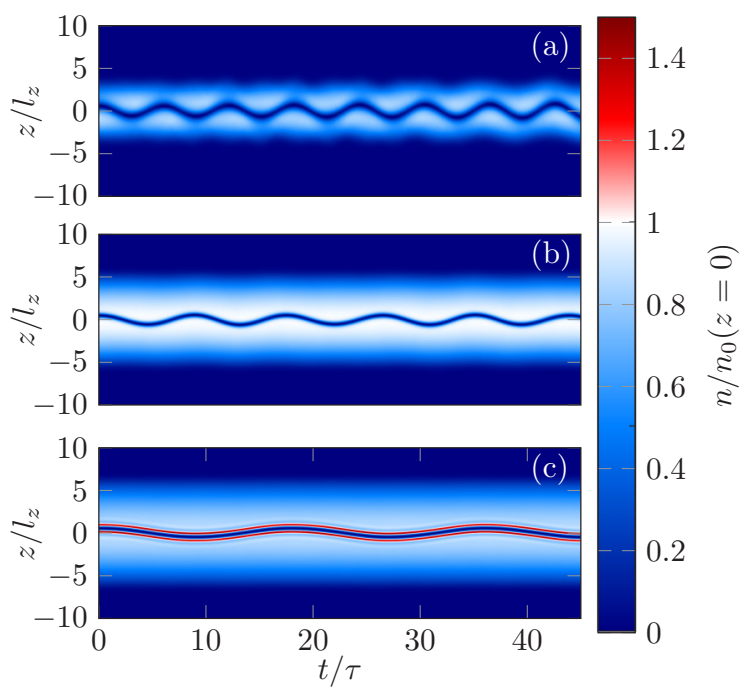

FIG. 2. Density dynamics of a dark soliton in the quasi-1D dipolar condensate for (a) $\varepsilon_{\mathrm{dd}}=-1.7$, (b) $\varepsilon_{\mathrm{dd}}=0$, and (c) $\varepsilon_{\mathrm{dd}}=-5.5$. These values correspond to close to $a_{\text {eff }}=0$, the nondipolar case, and close to the roton instability, respectively. Remaining parameters as in Fig. 1. Time is expressed in units of $\tau=1 / \omega_{z}$.

Note that this choice of offset gives $0.01<z_{0} / R_{z}<0.15$, that is, that the soliton is located within the central region of the condensate, away from the edges. Identical results are obtained by using the product of the background BEC and a traveling dark soliton solution from the uniform system [51,52]. Figure 2 shows three example cases with differing $\varepsilon_{\mathrm{dd}}$ (close to $a_{\mathrm{eff}}=0$, the nondipolar case $\varepsilon_{\mathrm{dd}}=0$, and close to a RI). Throughout, the soliton oscillates sinusoidally and stably through the BEC, with preserved form and oscillation amplitude. These stable oscillations exist throughout the stable regime of the background condensate. It is clear, however, that the oscillation period changes with $\varepsilon_{\mathrm{dd}}$ [even for condensates with similar sizes and curvatures, cf. Figs. 2(b) and 2(c)]. Furthermore, in Fig. 2(a) there is evidence that the solitonsound interactions are activating the dipole mode of the condensate, an effect which has been studied in nondipolar condensates [64]. To further explore the influence of dipolar interactions on the soliton oscillation frequency, Fig. 3 plots the oscillation frequency $\omega_{\mathrm{s}}$ of the soliton coordinate (defined as the point of minimum density) based on the 1D dipolar GPE for $|\beta|=61$ (red triangles) and 1500 (red circles). For $\varepsilon_{\mathrm{dd}}=0$ we recover the established result for the nondipolar system, $\omega_{\mathrm{s}} \approx \omega_{z} / \sqrt{2}$ [26]. More generally, $\omega_{\mathrm{s}}$ varies sensitively with $\varepsilon_{\mathrm{dd}}$, deviating by up to $60 \%$ from the nondipolar frequency. In comparison, for $\varepsilon_{\mathrm{dd}}=0$, the deviation from $\omega_{z} / \sqrt{2}$ is only significant in the very weakly interacting limit $\beta \lesssim 1$; for example, a nondipolar system with comparable condensate and soliton sizes to Fig. 2(a) oscillates to within $5 \%$ of $\omega_{z} / \sqrt{2}$. The scale of this sensitivity is surprising given that the other collective oscillations - the shape oscillations - in elongated dipolar BECs vary much more weakly with $\varepsilon_{\mathrm{dd}}$ [see, e.g., Fig. 11(a) of Ref. [65]].

In the Appendix we take steps to analytically describe the dependence of the soliton oscillation frequency on the interactions, following established techniques. Starting from 


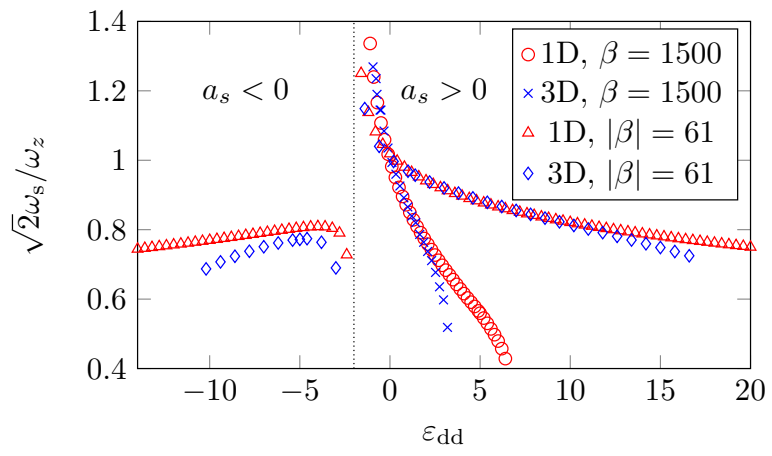

FIG. 3. Oscillation frequency of the dark soliton (starting as an off-center black soliton) based on the 1D dipolar GPE (red circles and triangles), 3D dipolar GPE (blue crosses and diamonds). The system parameters are as Fig. 1; the 3D system also assumes 164Dy atoms, $\omega_{\perp}=2 \pi \times 16 \mathrm{kHz}$ and $\left|a_{s}\right|=50 a_{0}$, where $a_{0}$ is the Bohr radius. The $3 \mathrm{D}$ system is stable only in the range of markers.

the approximation that the soliton wave function matches the nondipolar soliton, assuming that the soliton can be decomposed from the background fluid, and taking a local density approximation, we repeatedly derive the well-known result $\omega_{s}=\omega_{z} / \sqrt{2}$ for this system. We believe the inaccuracy of the local density approximation is due to the long-range interactions between the soliton and background fluid, negating the applicability of the separation of the two.

This discrepancy is not accounted for by the effective mass of the soliton: the denominator of $\sqrt{2}$ in the predicted nondipolar oscillation frequency is related to the soliton having an effective mass of $M_{\mathrm{s}}=2 \mathrm{~m}$. We have evaluated, and corrected for, the effective mass of the soliton, as per Ref. [52], and find no significant effect. The incapability of conventional methods describing solitons in terms of a particle model, so successful for nondipolar dark solitons, used to describe the observed oscillations leads us to conclude that the dipolar dark solitons are inherently extended and nonparticlelike excitations, which cannot be decomposed from the background BEC.

To assess the role of dimensionality, we have conducted the corresponding simulations using the full 3D dipolar GPE [66]. The dimensional stability of the dark solitons in this system is confirmed. Moreover, the 3D oscillation frequencies (blue crosses and diamonds in Fig. 3) are similar to the 1D results, although the RI regimes encroach to lower $\varepsilon_{\mathrm{dd}}$ in $3 \mathrm{D}$. For example, the 3D BEC is stable for $-10 \lesssim \varepsilon_{\mathrm{dd}} \lesssim-3$ for $a_{s}<0$ and $-2 \lesssim \varepsilon_{\mathrm{dd}} \lesssim 16$ for $a_{s}>0$. The decreased stability in 3D is due to the role of transverse magnetostriction in facilitating the RI [63]; indeed, as the ratio $\omega_{z} / \omega_{\perp}$ is decreased (system made more elongated), the RI is suppressed and approaches the $1 \mathrm{D}$ behavior.

Finally, we use 3D simulations to examine the dark solitons achievable in the elongated system of a recent experiment [44] with ${ }^{164}$ Dy atoms $\left(a_{\mathrm{dd}}=132 a_{0}\right), \theta=\pi / 2$, and $\left(\omega_{\perp}, \omega_{z}\right)=$ $2 \pi \times(128,2) \mathrm{Hz}$, with variations of $\varepsilon_{\mathrm{dd}}$ achieved through Feshbach tuning of $a_{s}$ [67] (this is distinct from our previous results where $\beta$ was fixed). Alongside introducing an off-center black soliton into the initial condition (as done so far), we also imprint a $\pi$-phase step in real time, akin to experimental engineering of dark solitons $[9,14]$. This generates a soliton plus

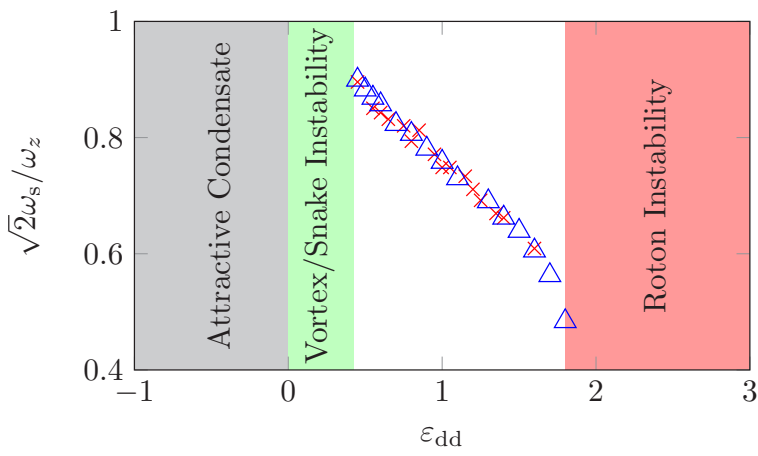

FIG. 4. Oscillation frequency and phase diagram for a dark soliton in a ${ }^{164}$ Dy BEC with Feshbach tuning of $a_{s}$, based on a recent experiment setup [44]. Shown are cases where the soliton is imposed in the initial condition (blue triangles, as per Fig. 1) and imprinted in real time (red crosses). Outside of the dark soliton regime, the condensate is either roton unstable (red), an attractive condensate incapable of supporting dark solitons (grey), or the dark solitons are dimensionally unstable. Parameters: $\theta=\pi / 2,\left(\omega_{\perp}, \omega_{z}\right)=$ $2 \pi \times(128,2) \mathrm{Hz}, a_{\mathrm{dd}}=132 a_{0}$, and $N=10000$.

sound waves. The oscillation frequency and phase diagram is depicted in Fig. 4. Stable dark solitons are supported for $0.4 \lesssim$ $\varepsilon_{\mathrm{dd}} \lesssim 1.8$; across this range $\omega_{\mathrm{s}}$ changes by a factor of 2 . Above this range, the RI arises, matching the observed experimental value of condensate collapse for this system. Below this range, the dark solitons undergo the snake instability into vortex rings. This is because the regime of small positive $\varepsilon_{\mathrm{dd}}$ corresponds to large positive $a_{s}$ and hence a small healing length; when this becomes smaller than the transverse system size, the condensate leaves the quasi-1D regime and becomes 3D in nature. For negative $\varepsilon_{\mathrm{dd}}$, i.e., negative $a_{s}$, the large and attractive contact interactions dominate to form a net attractive trapped condensate, in which dark solitons are not supported.

In conclusion, dark solitons are supported in trapped quasi-1D dipolar BECs, providing the background BEC is itself stable and net repulsively interacting. These excitations are accessible to current experiments. While dark solitons in nondipolar trapped BECs oscillate at a robust, characteristic ratio of the trap frequency, the oscillations in dipolar condensates become strongly dependent on the atomic interactions, and remarkably more sensitive than the collective surface modes. Analytical models derived from previously successful methods do not capture the interaction-dependent behavior. The dark soliton is strictly an extended excitation, not amenable to analytical treatment as a (local) particle, decomposable from the background. These states might offer a novel platform to study nonlocal dark solitons, to date observed in optics [68] and liquid crystals [69], with the immense control afforded by the atomic physics toolbox. Finally, our results show that this species of quantum canary [23] is particularly sensitive to the interactions, suggesting their use to probe the mesoscopic details of the quantum field, such as current open questions over quantum fluctuations in dipolar BECs [41,45,70,71].

Data supporting this publication is openly available under an 'Open Data Commons Open Database License' [72]. 


\section{ACKNOWLEDGMENTS}

T.B., M.E., and N.G.P. thank the Engineering and Physical Sciences Research Council (Grant No. EP/M005127/1) for support. K.P. and K.R. thank the National Science Center Grant No. DEC-2012/04/A/ST2/00090 for support.

\section{APPENDIX: SOLITON DYNAMICS}

In this appendix we extend the main analytical approaches for describing the particlelike motion of nondipolar dark solitons in trapped condensates to include dipolar interactions. The approach we consider follows that presented by Frantzeskakis [25] for the nondipolar case, which we adapt to include the effect of the nonlocal dipolar interactions. This approach begins by defining the "renormalized energy" of a dark soliton in a homogeneous dipolar system as the energy of the soliton state minus the energy of the nonsoliton state with the same number of particles, i.e., the energy associated with the soliton being present. Denoting the wave function featuring the dark soliton as $\psi_{s}$, the renormalized soliton energy is

$$
E_{s}=\int_{-\infty}^{\infty} d z\left[\frac{\hbar^{2}}{2 m}\left|\nabla \psi_{s}\right|^{2}+\hbar \omega_{\perp} a_{s} m f(z)^{2}\right]+E_{\mathrm{DDI}}
$$

where $f(z)=\left|\psi_{s}(z)\right|^{2}-n_{0}$ is the renormalized density, with $n_{0}$ being the density of the homogeneous soliton-free state. The renormalized energy contribution from the dipole-dipole interactions (written in momentum space for convenience) is

$$
\begin{aligned}
E_{\mathrm{DDI}} & =\frac{1}{2} \int_{-\infty}^{\infty} \int_{-\infty}^{\infty} d z d z^{\prime} f(z) U_{\mathrm{int}}\left(z-z^{\prime}\right) f\left(z^{\prime}\right) \\
& =\frac{1}{4 \pi} \int_{-\infty}^{\infty} d k \tilde{f}(k) \tilde{U}_{\mathrm{int}}(k) \tilde{f}(-k),
\end{aligned}
$$

where the tilde denotes the Fourier transform.

We approximate the wave function of the dipolar dark soliton with the wave function of the (nondipolar) Shabat-Zakharov dark soliton for a uniform gas, given by

$$
\psi_{\mathrm{s}}(z)=\sqrt{n_{0}}\left[\beta_{v} \tanh \left(\frac{z-z_{0}}{\xi} \beta_{v}\right)+i \sqrt{1-\beta_{v}^{2}}\right] e^{-i \mu t / \hbar},
$$

with $\beta_{v}=\sqrt{1-v^{2} / c^{2}}$, where $v$ is the velocity of the soliton, $\mu=n_{0} g$ is the chemical potential of the background condensate, $c=\sqrt{\mu / m}$ is the speed of sound, $z_{0}$ is the initial position of the soliton, and $\xi=\hbar / \sqrt{m \mu}$ is the condensate's healing length (which characterizes the soliton size).

As reported in Refs. [51,52], away from the roton and phonon instabilities, the true dipolar dark soliton of the homogeneous system closely matches the (nondipolar) Shabat-Zahharov soliton (A3) with the modified scattering length $a_{\text {eff }}$ [as defined in Eq. (3) of the main paper]. Within the local density approximation, these effective contact interactions account for the effect of the dipolar interactions on the healing length and speed of sound of the system, which in turn specify the absolute size and speed of the soliton. Thus we proceed by approximating the dipolar dark soliton solution as an ansatz given by the Zakharov-Shabat solution (A3) but with $a_{\mathrm{s}} \mapsto a_{\mathrm{eff}}$. The mapping of the homogeneous chemical potential follows as $\mu=2 \hbar \omega_{\perp} n_{0} a_{s} \mapsto \mu=2 \hbar \omega_{\perp} n_{0} a_{s}[1-$ $\left.\frac{1}{4} \varepsilon_{\mathrm{dd}}(1+3 \cos 2 \theta)\right]$.

For this ansatz the Fourier transform of the renormalized density, $\tilde{f}(k)$, is a function which decreases exponentially with momentum as $1 / \xi$. Precisely, in our convention of the Fourier transform, $\tilde{f}(k)=-\pi k \xi^{2} n_{0} \operatorname{cosech}\left(\frac{k \pi \xi}{2 \beta_{v}}\right) e^{i z_{0} k}$. The Fourier transform of the dipolar potential $\tilde{U}_{\text {int }}(k)$ on the other hand is a function decreasing as $1 / l_{\perp}$. Dimensional stability of the dark soliton requires $l_{\perp}<\xi$. Thus in the integral (A2) one can approximate $\tilde{U}_{\mathrm{dd}}(k)$ with its expansion around $k=0$,

$$
\begin{aligned}
\tilde{U}_{\mathrm{dd}}(k) & =4 U_{0} l_{\perp}\left(q e^{q} E_{1}[q]-\frac{1}{3}\right) \\
& \approx-4 U_{0} l_{\perp}\left(q[\gamma+\ln (q)]+\frac{1}{3}\right),
\end{aligned}
$$

where $q=k^{2} l_{\perp}^{2} / 2, U_{0}=C_{\mathrm{dd}}(1+3 \cos 2 \theta) / 32 \pi l_{\perp}^{3}$ and $\gamma \approx$ 0.56 is the Euler-Mascheroni constant. Within these approximations, the renormalized energy of the dipolar dark soliton in a homogeneous system as per Eq. (A1) is

$$
\begin{aligned}
E_{s}= & \frac{4}{3} n_{0} \hbar c \beta_{v}^{3}-\frac{C_{\mathrm{dd}} n_{0}^{2}(1+3 \cos 2 \theta)}{2 \pi^{5} \xi} \\
& \times\left\{\frac{\pi^{4}}{15}\left[\gamma+\ln \left(\frac{2 \beta_{v}^{2} l_{\perp}^{2}}{\pi^{2} \xi^{2}}\right)\right]+\mathcal{K}_{4}\right\} \beta_{v}^{5},
\end{aligned}
$$

where $\mathcal{K}_{n}=\int_{-\infty}^{\infty} d u u^{n} \ln \left(u^{2}\right) \operatorname{cosech}^{2} u$ and $\mathcal{K}_{4} \approx 9.73$. In the absence of dipoles $\left(C_{\mathrm{dd}}=0\right)$ this reduces to the well-established result $E_{s}=\frac{4}{3} n_{0} \hbar c \beta_{v}^{3}[24,25]$. The second, additional term accounts for the dipolar interactions.

Now, to extend this prediction for the soliton energy from a homogeneous condensate to a trapped condensate, we can adopt the method described by Frantzeskakis [25,73] in which Eq. (A5) is assumed to hold locally for a background density which varies slowly in space $n(z)$. We replace all density dependence in Eq. (A5) with the varying density of the harmonically trapped condensate. We write this density analytically in the Thomas-Fermi limit as $n(z)=n_{0}-m \omega_{z}^{2} z^{2} / 4 \hbar \omega_{\perp} a_{\mathrm{eff}}$, with $a_{\text {eff }}$ defined in the main paper, and make the replacement $n_{0} \mapsto n(z)$, including $c \mapsto c(z)$ and $\xi \mapsto \xi(z)$, in Eq. (A5).
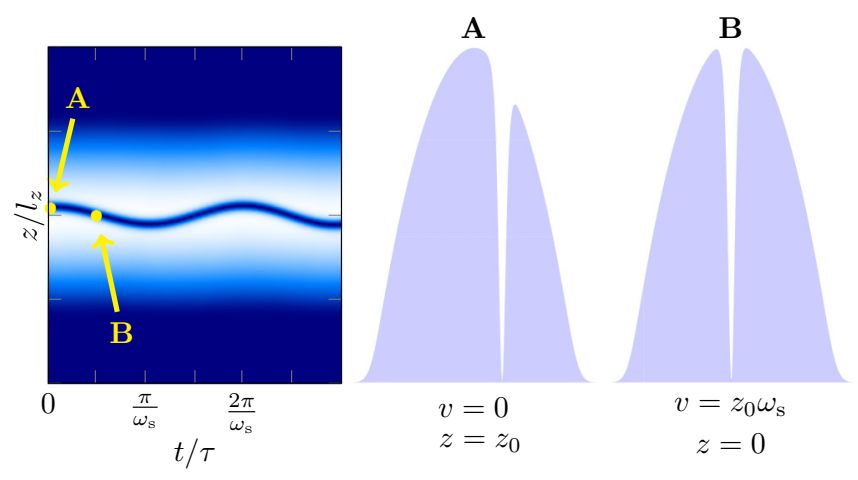

FIG. 5. Left: Density of the atomic cloud with soliton as a function of time and position. Right: Schematic cut through density profile at two instances of times: $t=0$, situation A, when the soliton is displaced from the trap center by $z_{0}$ and it has a zero velocity, and $t=\pi /\left(2 \omega_{\mathrm{s}}\right)$, situation $\mathrm{B}$, when the soliton is at a quarter of period, passing the center of the trap with the velocity $\omega_{\mathrm{s}} z_{0}$. 
Then, expanding the equation in the limit of slow solitons and after some manipulation, we write the result in the form

$$
E(z, v)=E_{0}+\frac{1}{2} m_{s} v^{2}+\frac{1}{4} m_{s} \omega_{z}^{2} z^{2} .
$$

This describes the soliton as a particle with effective mass $m_{s}$ and rest mass energy $E_{0}$. The effective mass is given by

$$
m_{s}=-\sqrt{\frac{2 n_{0}}{a_{\mathrm{s}}}} \frac{l_{\perp} m}{\left[1-\frac{1}{4} \varepsilon_{\mathrm{dd}}(1+3 \cos 2 \theta)\right]}+\frac{5 C_{\mathrm{dd}}(1+3 \cos 2 \theta) n_{0}^{3 / 2}}{4 \sqrt{2} \pi^{5} l_{\perp}^{3} \omega_{\perp}^{2} a_{\mathrm{s}}^{1 / 2}\left[1-\frac{1}{4} \varepsilon_{\mathrm{dd}}(1+3 \cos 2 \theta)\right]^{2}}\left\{\frac{\pi^{4}}{15}\left[\gamma+\frac{2}{5}+\ln \left(\frac{4 n_{0} a_{s}}{\pi^{2}}\right)\right]+\mathcal{K}_{4}\right\} .
$$

and the rest mass energy is

$$
E_{0}=\frac{4}{3}\left(\frac{2 \hbar^{3} n_{0}^{3} a_{\mathrm{s}} \omega_{\perp}}{m\left[1-\frac{1}{4} \varepsilon_{\mathrm{dd}}(1+3 \cos 2 \theta)\right]^{2}}\right)^{1 / 2}-\frac{C_{\mathrm{dd}}(1+3 \cos 2 \theta) n_{0}^{5 / 2} a_{\mathrm{s}}^{1 / 2}}{\sqrt{2} \pi^{5} l_{\perp}\left[1-\frac{1}{4} \varepsilon_{\mathrm{dd}}(1+3 \cos 2 \theta)\right]^{2}}\left\{\frac{\pi^{4}}{15}\left[\gamma+\ln \left(\frac{4 n_{0} a_{s}}{\pi^{2}}\right)\right]+\mathcal{K}_{4}\right\} .
$$

This result tells us that the dipolar dark soliton behaves as a nondipolar dark soliton with dipolar effective mass and predicts the soliton oscillation frequency to be

$$
\omega_{\mathrm{s}}=\frac{\omega_{z}}{\sqrt{2}} .
$$

This incorrect prediction tells us that the observed soliton oscillation frequency is not obtainable from the energy with the assumptions used. Note that if the true (numerically obtained) dipolar dark soliton solution is employed, then the soliton energy, Eq. (A1), gives close agreement with the prediction of Eq. (A6), in the appropriate limit (small soliton oscillation amplitude, low speed, and a Thomas-Fermi-like background condensate).

We further explore the role of the local density approximation through the law of conservation of energy, removing the assumption of a stationary soliton from the previous analysis. We compare the energy of a soliton at two instances in time, denoted in Fig. 5 with A and B. Situation A corresponds to the initial condition, $t=0$, when the soliton is at position $z=z_{0}$ with $v=0$. Then the soliton accelerates in the harmonic potential and situation $B$ is reached when the soliton passes the center of the trap; then its position is $z=0$ with the maximal velocity. We observed numerically that the soliton is moving periodically in the potential (see example in the left panel of Fig. 5). Hence, at situation B, the velocity of the soliton should be equal to $z_{0} \omega_{\mathrm{s}}$. In what follows we rewrite the estimation of the energy, Eq. (A6), in terms of the chemical potential and velocity of a dipolar soliton:

$$
\begin{aligned}
E(\mu, v)= & A_{0}\left(\mu-m v^{2}\right)^{3 / 2}+A_{1}\left(\mu-m v^{2}\right)^{5 / 2} \\
& +B_{1}\left(\mu-m v^{2}\right) \ln \left(\frac{\mu-m v^{2}}{\hbar \omega_{\perp}}\right) .
\end{aligned}
$$

The exact form of the coefficients $A_{0}, A_{1}$, and $B_{1}$ do not play any role in the derivation below. If we assume the Thomas-Fermi profile of the gas, then $\mu(z)=\mu_{0}\left(1-z^{2} / R_{z}^{2}\right)$, where $R_{z}=\frac{3}{2} \frac{N \tilde{g}}{\hbar \omega_{z}}, \mu_{0}=\frac{3 N \tilde{g}}{4 R_{z}}$, and $\tilde{g}=2 \hbar \omega_{\perp} a_{s}\left[1-\frac{1}{4} \varepsilon_{\mathrm{dd}}(1+\right.$ $3 \cos 2 \theta)]$.

The energies in both situations, A and B, read

$$
\begin{aligned}
E_{A}= & E\left(\mu\left(z_{0}\right), v=0\right) \\
= & A_{0}\left[\mu\left(z_{0}\right)\right]^{\frac{3}{2}}+A_{1}\left[\mu\left(z_{0}\right)\right]^{\frac{5}{2}}+B_{1}\left[\mu\left(z_{0}\right)\right] \ln \left(\frac{\mu\left(z_{0}\right)}{\hbar \omega_{\perp}}\right), \\
E_{B}= & E\left(\mu_{0}, v=z_{0} \omega_{\mathrm{s}}\right) \\
= & A_{0}\left[\mu_{0}-m\left(z_{0} \omega_{\mathrm{s}}\right)^{2}\right]^{\frac{3}{2}}+A_{1}\left[\mu_{0}-m\left(z_{0} \omega_{\mathrm{s}}\right)^{2}\right]^{\frac{5}{2}} \\
& +B_{1}\left[\mu_{0}-m\left(z_{0} \omega_{\mathrm{s}}\right)^{2}\right] \ln \left(\frac{\mu_{0}-m\left(z_{0} \omega_{\mathrm{s}}\right)^{2}}{\hbar \omega_{\perp}}\right) .
\end{aligned}
$$

The comparison between both energies is done only in the limit $\epsilon=z_{0} / R_{z} \ll 1$. So for solitons relatively close to the trap center, we expand the identity $E_{A}=E_{B}$ to the second order of $\epsilon$. Simple algebra gives

$$
\omega_{\mathrm{s}}^{2}=\frac{\mu_{0}}{m R_{z}^{2}} \Rightarrow \omega_{\mathrm{s}}^{2}=\frac{\omega_{z}^{2}}{2} .
$$

The derivation is based on the following approximations:

(1) Approximate formula for the soliton energy,

(2) Local density approximation, and

(3) Expansion with respect to the small parameter $z_{0} / R_{z}$.

The last approximation seems to be very reasonable, even for the gas with van der Waals interaction, only we expect that the sinusoidal oscillation with frequency $\omega_{z} / \sqrt{2}$ is valid for solitons localized close to the center of the trap.
[1] P. Emplit, J. P. Hamaide, F. Reynaud, C. Froehly, and A. Barthelemy, Opt. Commun. 62, 374 (1987).

[2] D. Krökel, N. J. Halas, G. Giuliani, and D. Grischkowsky, Phys. Rev. Lett. 60, 29 (1988).

[3] A. M. Weiner, J. P. Heritage, R. J. Hawkins, R. N. Thurston, E. M. Kirschner, D. E. Leaird, and W. J. Tomlinson, Phys. Rev. Lett. 61, 2445 (1988).

[4] P. K. Shukla and B. Eliasson, Phys. Rev. Lett. 96, 245001 (2006).
[5] R. Heidemann, S. Zhdanov, R. Sütterlin, H. M. Thomas, and G. E. Morfill, Phys. Rev. Lett. 102, 135002 (2009).

[6] A. Chabchoub, O. Kimmoun, H. Branger, N. Hoffmann, D. Proment, M. Onorato, and N. Akhmediev, Phys. Rev. Lett. 110, 124101 (2013).

[7] M. Chen, M. A. Tsankov, J. M. Nash, and C. E. Patton, Phys. Rev. Lett. 70, 1707 (1993). 
[8] S. Burger, K. Bongs, S. Dettmer, W. Ertmer, K. Sengstock, A. Sanpera, G. V. Shlyapnikov, and M. Lewenstein, Phys. Rev. Lett. 83, 5198 (1999).

[9] J. Denschlag, J. E. Simsarian, D. L. Feder, C. W. Clark, L. A. Collins, J. Cubizolles, L. Deng, E. W. Hagley, K. Helmerson, W. P. Reinhardt, S. L. Rolston, B. I. Schneider, and W. D. Phillips, Science 287, 97 (2000).

[10] Z. Dutton, M. Budde, C. Slowe, and L. V. Hau, Science 293, 663 (2001).

[11] B. P. Anderson, P. C. Haljan, C. A. Regal, D. L. Feder, L. A. Collins, C. W. Clark, and E. A. Cornell, Phys. Rev. Lett. 86, 2926 (2001).

[12] G.-B. Jo, J.-H. Choi, C. A. Christensen, T. A. Pasquini, Y.-R. Lee, W. Ketterle, and D. E. Pritchard, Phys. Rev. Lett. 98, 180401 (2007).

[13] P. Engels and C. Atherton, Phys. Rev. Lett. 99, 160405 (2007).

[14] C. Becker, S. Stellmer, P. Soltan-Panahi, S. Dörscher, M. Baumert, E.-M. Richter, J. Kronjäger, K. Bongs, and K. Sengstock, Nat. Phys. 4, 496 (2008).

[15] A. Weller, J. P. Ronzheimer, C. Gross, J. Esteve, M. K. Oberthaler, D. J. Frantzeskakis, G. Theocharis, and P. G. Kevrekidis, Phys. Rev. Lett. 101, 130401 (2008).

[16] S. Stellmer, C. Becker, P. Soltan-Panahi, E.-M. Richter, S. Dörscher, M. Baumert, J. Kronjäger, K. Bongs, and K. Sengstock, Phys. Rev. Lett. 101, 120406 (2008).

[17] J. J. Chang, P. Engels, and M. A. Hoefer, Phys. Rev. Lett. 101, 170404 (2008).

[18] I. Shomroni, E. Lahoud, S. Levy, and J. Steinhauer, Nat. Phys. 5, 193 (2009).

[19] C. Hamner, J. J. Chang, P. Engels, and M. A. Hoefer, Phys. Rev. Lett. 106, 065302 (2011).

[20] L. M. Aycock, H. M. Hurst, D. Genkina, H.-I. Lu, V. Galitski, and I. B. Spielman, Proc. Natl. Acad. Sci. USA 114, 2503 (2017).

[21] I. Bloch, J. Dalibard, and W. Zwerger, Rev. Mod. Phys. 80, 885 (2008).

[22] K. Henderson, C. Ryu, C. MacCormick, and M. G. Boshier, New J. Phys. 11, 043030 (2009).

[23] J. Anglin, Nat. Phys. 4, 437 (2008).

[24] Y. S. Kivshar and B. Luther-Davies, Phys. Rep. 298, 81 (1998).

[25] D. J. Frantzeskakis, J. Phys. A 43, 213001 (2010).

[26] T. Busch and J. R. Anglin, Phys. Rev. Lett. 84, 2298 (2000).

[27] V. V. Konotop and L. P. Pitaevskii, Phys. Rev. Lett. 93, 240403 (2004).

[28] V. A. Brazhnyi, V. V. Konotop, and L. P. Pitaevskii, Phys. Rev. A 73, 053601 (2006).

[29] D. E. Pelinovsky, D. J. Frantzeskakis, and P. G. Kevrekidis, Phys. Rev. E 72, 016615 (2005).

[30] D. J. Frantzeskakis, and P. G. Kevrekidis, and N. P. Proukakis, Phys. Lett. A 364, 129 (2007).

[31] D. E. Pelinovsky and P. G. Kevrekidis, AMS Contemp. Math 473, 159 (2008).

[32] G. Huang, J. Szeftel, and S. Zhu, Phys. Rev. A 65, 053605 (2002).

[33] G. Theocharis, P. G. Kevrekidis, M. K. Oberthaler, and D. J. Frantzeskakis, Phys. Rev. A 76, 045601 (2007).

[34] A. M. Kamchatnov and M. Salerno, J. Phys. B: At. Mol. Opt. 42, 185303 (2009).

[35] G. E. Astrakharchik and L. P. Pitaevskii, Europhys. Lett. 102, 30004 (2013).
[36] A. Griesmaier, J. Werner, S. Hensler, J. Stuhler, and T. Pfau, Phys. Rev. Lett. 94, 160401 (2005).

[37] Q. Beaufils, R. Chicireanu, T. Zanon, B. Laburthe-Tolra, E. Maréchal, L. Vernac, J. C. Keller, and O. Gorceix, Phys. Rev. A 77, 061601(R) (2008).

[38] M. Lu, N. Q. Burdick, S. H. Youn, and B. L. Lev, Phys. Rev. Lett. 107, 190401 (2011).

[39] Y. Tang, N. Q. Burdick, K. Baumann, and B. L. Lev, New J. Phys. 17, 045006 (2015).

[40] K. Aikawa, A. Frisch, M. Mark, S. Baier, A. Rietzler, R. Grimm, and F. Ferlaino, Phys. Rev. Lett. 108, 210401 (2012).

[41] L. Chomaz, S. Baier, D. Petter, M. J. Mark, F. Wächtler, L. Santos, and F. Ferlaino, Phys. Rev. X 6, 041039 (2016).

[42] T. Lahaye, C. Menotti, L. Santos, M. Lewenstein, and T. Pfau, Rep. Prog. Phys. 72, 126401 (2009).

[43] H. Kadau, M. Schmitt, M. Wenzel, C. Wink, T. Maier, I. FerrierBarbut, and T. Pfau, Nature 530, 194 (2016).

[44] I. Ferrier-Barbut, H. Kadau, M. Schmitt, M. Wenzel, and T. Pfau, Phys. Rev. Lett. 116, 215301 (2016).

[45] M. Schmitt, M. Wenzel, F. Böttcher, I. Ferrier-Barbut, and T. Pfau, Nature 539, 259 (2016).

[46] C. Chin, R. Grimm, P. Julienne, and E. Tiesinga, Rev. Mod. Phys. 82, 1225 (2010).

[47] S. Giovanazzi, A. Gorlitz, and T. Pfau, Phys. Rev. Lett. 89, 130401 (2002).

[48] F. Deuretzbacher, J. C. Cremon, and S. M. Reimann, Phys. Rev. A 81, 063616 (2010); 87, 039903(E) (2013).

[49] S. Sinha and L. Santos, Phys. Rev. Lett. 99, 140406 (2007).

[50] V. E. Zakharov and A. B. Shabat, Zh. Eksp. Teor. Fiz. 64, 1627 (1973) [Sov. Phys. JETP 37, 823 (1973)].

[51] T. Bland, M. J. Edmonds, N. P. Proukakis, A. M. Martin, D. H. J. O'Dell, and N. G. Parker, Phys. Rev. A 92, 063601 (2015).

[52] M. J. Edmonds, T. Bland, D. H. J. O'Dell, and N. G. Parker, Phys. Rev. A 93, 063617 (2016).

[53] K. Pawłowski and K. Rzażewski, New J. Phys. 17, 105006 (2015).

[54] J. Cuevas, B. A. Malomed, P. G. Kevrekidis, and D. J. Frantzeskakis, Phys. Rev. A 79, 053608 (2009).

[55] S. K. Adhikari, Phys. Rev. A 90, 055601 (2014).

[56] B. B. Baizakov, S. M. Al-Marzoug, and H. Bahlouli, Phys. Rev. A 92, 033605 (2015).

[57] B. A. Umarov, N. A. B. Aklan, B. B. Baizakov, and F. Kh. Abdullaev, J. Phys. B: At. Mol. Opt. Phys. 49, 125307 (2016)

[58] M. J. Edmonds, T. Bland, R. Doran, and N. G. Parker, New J. Phys. 19, 023019 (2017).

[59] S. K. Adhikari, Phys. Rev. A 89, 043615 (2014).

[60] C. Menotti and S. Stringari, Phys. Rev. A 66, 043610 (2002).

[61] N. G. Parker and D. H. J. O’Dell, Phys. Rev. A 78, 041601(R) (2008).

[62] Y. Cai, M. Rosenkranz, Z. Lei, and W. Bao, Phys. Rev. A 82, 043623 (2010).

[63] J. L. Bohn, R. M. Wilson, and S. Ronen, Laser Phys. 19, 547 (2009).

[64] N. G. Parker, N. P. Proukakis, M. Leadbeater, and C. S. Adams, Phys. Rev. Lett. 90, 220401 (2003).

[65] R. M. W. van Bijnen, N. G. Parker, S. J. J. M. F. Kokkelmans, A. M. Martin, and D. H. J. O’Dell, Phys. Rev. A 82, 033612 (2010) 
[66] V. Lonar, A. Balaz, A. Bogojevic, S. Skrbic, P. Muruganandam, and S. K. Adhikari, Comput. Phys. Commun. 200, 406 (2016); R. Kishor Kumar, L. E. Young-S., D. Vudragovic, A. Balaz, P. Muruganandam, and S. K. Adhikari, ibid. 195, 117 (2015).

[67] Y. Tang, A. G. Sykes, N. Q. Burdick, J. M. DiSciacca, D. S. Petrov, and B. L. Lev, Phys. Rev. Lett. 117, 155301 (2016).

[68] A. Dreischuh, D. Neshev, D. E. Petersen, O. Bang, and W. Krolikowski, Phys. Rev. Lett. 96, 043901 (2006).
[69] A. Piccardi, A. Alberucci, N. Tabiryan, and G. Assanto, Opt. Lett. 36, 1356 (2011).

[70] R. N. Bisset, R. M. Wilson, D. Baillie, and P. B. Blakie, Phys. Rev. A 94, 033619 (2016).

[71] F. Wächtler and L. Santos, Phys. Rev. A 94, 043618 (2016).

[72] Newcastle University Data, http://dx.doi.org/10.17634/1371394.

[73] P. O. Fedichev, A. E. Muryshev, and G. V. Shlyapnikov, Phys. Rev. A 60, 3220 (1999). 